\title{
Proposal for Standardization of Green Information Centric Networking Based Communication Utilizing Proactive Caching in Intelligent Transport System
}

\author{
Quang N. Nguyen ${ }^{1}$, M. Arifuzzaman ${ }^{2, *}$, D. Zhang ${ }^{1, *}$, \\ K. $\mathrm{Yu}^{1}$ and T. Sato ${ }^{1, * *}$ \\ ${ }^{1}$ Faculty of Science and Engineering, Waseda University, Tokyo, Japan \\ ${ }^{2}$ Department of Electronics and Communications Engineering, East West University \\ * Student member, IEEE \\ ** Fellow, IEEE \\ E-mail: \{quang.nguyen; arif; di_zhang; yukeping\}@fuji.waseda.jp, \\ t-sato@waseda.jp
}

Received 7 March 2016;

Accepted 8 June 2016

\begin{abstract}
Nowadays, Internet is mostly used to access content and users are also interested in content, instead of location. Information-Centric Networking (ICN) has drawn substantial consideration over past few years because its appearance introduced a new potential Internet architecture (deploying extensive cache structure to distribute and deliver information). Though ICN has undoubtedly become a promising Future Internet architecture to solve current host-centric Internet problems, conventional ICN design still has its own drawbacks. These include caching, routing, etc., particularly mobility issue and energy-efficiency problem due to additional energy consumption for Content Routers' caching capability. In order to address the energy flaw and enhance the mobility performance of ICN Architecture, in this article, we have designed, evaluated and proposed a wireless Information Centric Networking (ICN) based access model within the context of Intelligent Transport System (ITS) for ICN standardization procedure. We take into consideration the fact
\end{abstract}

Journal of ICT, Vol. 4_l, 35-64.

doi: $10.13052 /$ jicts2245-800X.413

(c) 2016 River Publishers. All rights reserved. 
that number of mobile devices increases rapidly day by day as well as the public transportation (e.g. train, bus, etc) becomes more popular nowadays. Besides, mobile users may ask for different content interests via Internet during the period of time when they are on public transport vehicle. We then propose a wireless communication topology in ICN for the ITS by utilizing the smart scheduling algorithm with proactive-caching based strategy to deal with possible and practical situations of commuters (mobile content users). In our scheme, the dedicated content provider proactively sends appropriate segments of requested content (based on chunk-level structure of content) to expected proximity Aggregation content nodes located at neighbor stations (future attachment points) on the moving path of public transportation vehicle. This working scheme substantially enhances the mobile user's quality of experience and network performance in ICN. Moreover, the proposed ICN based system adapts the network nodes to their optimized network utilization values according to content popularity by conducting cross-layer optimization. This mechanism provides mobile users more reliable and effective Green Information and Communication Systems (ICS) when they travel with public transportation. Therefore, the proposal integrates Green networking into ICN by combining the benefits of innovated rate-adaptivity and proactive-caching based schemes for achieving highly scalable, reliable and energy-efficient network performance in future transportation Information-centric communication system with data-awareness. In addition, we identify that there are currently various ICN-based models and emphasize the need of an official ICN international standard for wireless communication in general and transportation system in particular. Following, by evaluating the proposal, we show that the proposed cross-layer wireless content access model in ICN for ITS is a promising and feasible contribution for the standardization process of ICN.

Keywords: Information Centric Networking (ICN), Standardization, Future Internet (FI), Intelligent Transportation Systems (ITS), Green Networking, Wireless Access Scheme, Next Generation Mobile Communication.

\section{Introduction}

Internet has becomes an essential infrastructure for our society since it plays an important part for all industries, economics and social activities of our daily lives. Today, we have to face with Big Data issue, and this leads to content access, which is a challenging issue due to bandwidth limitation and 
exponential Internet growth (especially in case of real-time access and highresolution content). This problem gets more and more challenging because our current host-centric Internet model has various problems including: security, content distribution, mobility and scalability (scale of network, in terms of users, devices and traffic). This leads to an Internet paradigm shift trend to match the key requirements of Future Internet (FI): from remote resource access to information access and change of traffic usage from host-to-host (location centric) network into Information Centric Networking (ICN) model $[1,2]$. Thanks to its innovated working mechanism, ICN has become a pioneer for the Future Internet architecture to solve current host-centric Internet's serious problems of inefficiencies, security, then brings benefits to all the stakeholders.

In essence, ICN architecture [2, 3] can handle mobility more efficiently since the content can be found en-route even after the handoff and especially better support for the scope of location transparency, i.e. the mobile node can change its Point of Attachment (PoA) and still maintain the consistent name for the user mobile. However, supporting smooth connection for seamless content retrieval is still a major issue and ICN mobility concern has not been received adequate exploration despite of the fact that wireless technologies is becoming a popular means for Internet users to get information/data. This issue becomes more critical for mobile user in the case of wireless communication in transportation system because the period of time a transportation vehicle stops at a station is relatively short, then the Point of Attachment (PoA) may be changed before the content user gets satisfied content data. Moreover, wireless access scheme has limited bandwidth and it is a challenge to access content in real-time for interactive services.

Therefore, in order to address the ICN architecture's mobility problem and enhance the performance, effectiveness along with energy efficiency of ICN in case of transportation systems' wireless access, the aim of this work is building a proactive-cache based flexible ICN architecture to support the seamless wireless communication with energy saving for the Intelligent Transport System (ITS). By considering the different practical scenarios (e.g. the case that mobile users can leave the public transport vehicle earlier than expected) to prevent possible unnecessary content traffic, reduce congestion and energy consumption as well as considering low-cost feature of Wifi technology, we do believe our proposal will become a feasible and efficient pioneer solution to on-going ICN standardization procedure for utilizing in transportation industry. 
The contributions of this proposal are follows:

- We take into consideration the widespread feature of public transportation with predictable routes and the fact that commuters tend to use mobile devices during period when they are on public transportation in modern digital society.

- We integrate both green networking and innovated proactive-caching based scheme in ICN mobility together to raise energy efficiency (about $20 \%$ energy-saving compared to current designs) and effectiveness (lower latency and higher data rate) for the goal of green and seamless mobility in ICN.

- Mathematical energy models for various network systems are formulated to evaluate the energy efficiency of the proposal. Then, evaluation and comparison of energy consumption and key performance metrics between proposed model and existing network designs (traditional IPbased network and Conventional ICN model) are conducted through simulation.

- The proposal aims at energy-efficiency issue in ICN in the context that energy efficiency has not been stated clearly in current ICN standardization procedure of major international standardization bodies including: ITU, IETF and IRTF. In addition, this issue is getting more critical in the era of IoT in the case of ICN due to the fact that ICN needs additional power consumption for caching energy.

As a result, the proposal acts as a potential ICN standardization approach which integrates the benefit of Green networking into ICN. In particular, from this work, the transportation industry (transportation companies) can offer customers smooth, robust and secured connection transparently during periods when they stay on a transport vehicle with lower energy cost.

The rest of this article is organized as follows: In Section 2, we state the related works. We point out current major ICN projects and our research motivation for transportation communication standardization in ICN in Section 3. Our proposed ITS model together with its proactive ICN caching strategy and smart scheduler mechanism are elaborated in Section 4. Section 5 describes the Adaptive Link Rate operating strategy for greening proposed ICN-based ITS. The energy model for our proposal and current IP based network architecture as well as conventional ICN design are clarified in Section 6. Then, we present simulation results and discussion for the energy-efficiency and effectiveness of the proposal architecture in Section 7. Finally, Section 8 concludes our article with a summary and present some directions for our future work. 


\section{Related Work}

Nowadays, mobility content access in real time has become a challenging issue due to bandwidth limitation and exponential Internet growth.

Pre-caching/proactive caching is recognized as one of the major schemes to reduce the response time, latency and enhance the user experience. Thus, a number of works have been conducted on proactive caching schemes and prefetching technique for the current client-server Internet architecture to make a desired data objects immediately available to a mobile user when the mobile moves to a new network attachment point. Regarding the proactive caching approaches for mobility, in [4], all neighboring proxies caching content items are initiated to match the mobile's subscriptions after the occurrence of a disconnection. Then, when the mobile node connects to one of these proxies, it can immediately get contents which were already transmitted to that proxy at the time of its disconnection. A selective neighbor caching (SNC) scheme is stated in [5] to reduce signaling overhead and handover delay in WLAN. The authors introduced a predefined threshold value of handoff probability considering handoff frequencies between APs (Access Points) to select neighbor APs for their SNC model. Another selective neighbor caching scheme is exploited for enhancing seamless mobility in ICN as defined in [6]. In the proposal, an optimized subset of neighbor proxies are selected as a pre-fetching destination of the content and the mobility behavior of users is considered to select the prospective neighbors. However, the proactive caching solutions in existing mobile network infrastructure are different from respective approaches in ICN. Since in general, the mobile host's context is proactive utilized in current system, whereas the goal of ICN system is only offering the mobile nodes content that match their interests.

For Green networking, Adaptive Link Rate (ALR) [7] is one of the wellknown techniques to reduce the power consumption of network systems by dynamically varying link rate to its utilization, hence response to link utilization quickly. A real-time hardware-prototype ALR system is implemented and analyzed in [8] as a practical way to evaluate the realtime performance of ALR, instead of simulation. A detailed survey of various Green communication applying ALR mechanism is presented in [9]. Besides, a combination of two greening approaches (rate-adaptivity and power-aware routing) is stated in [10] to save network power consumption by giving a simple model of power based on two kinds of link states (on and off), then compare the benefit of each one. Though ALR technique is mainly applied to IP-based architecture at this moment, we consider its novel working mechanism is also 
useful for the future Internet architecture (ICN). Since power consumption of network system can be taken as a function of network link load, then adapting network links to a rate which is proportional to the content interest traffic can save considerable amount of energy (as shown in our previous work for wired-connection network model [11]).

In addition, some previous researches also deal with mobility issues of mobile nodes in transportation system. For example, the authors of [12] proposed a commuter router infrastructure for public transportation system, but their scheme is mainly equipping additional routers with store-and-forward rather than maintaining an uninterrupted connection via Internet. In [13], Worldwide interoperability for microwave access (WiMAX) is considered for communication in railway scenario because of its QoS and mobility support at high speed. However, WIMAX technology has its own drawbacks and is insufficient for long-term deployment. Researchers in [14] built architecture deploying multiple cooperatively operating access routers on the train. This approach improves network performance but it requires additional coordination mechanism and endures higher overhead. Recently, a few researches also deal with this scope in ICN. A vehicle-to-vehicle communication in ICN is stated in [15] by extending the Content-Centric Networking (CCN) framework to support content delivery for vehicular communication efficiently. In [16], a prototype of energy-efficient and reliable ICN based wireless communication technology within the context of ITS is proposed for ICN standardization process. The solution combines both green networking and innovated proactive-caching based scheme in ICN mobility together to raise energy efficiency and effectiveness for the goal of green and seamless mobility in ICN.

\section{Major ICN Models and Research Motivation for Transportation Communication Standardization in ICN}

There are multiple recent works based on state-of-the-art ICN to realize the innovated mechanism of Information-Centric Internet for the Future Internet (FI) architecture, and the most notable works are: NDN (Named Data Networking) [17], PSIRP (Publish/Subscribe Internet Routing Paradigm) [18] and Network-Inf (Network of Information) [19]. NDN is a prototype of ICN and focuses on retrieving contents by its name instead of network's locations or addresses $[1,3]$. This mechanism is different from current host-to-host Internet model, which is not a scalable solution for high volume network traffic. NDN 
Naming scheme is hierarchical and human-readable. NDN also has Contentbased security with built-in Data packet security. Different from NDN, in PSIRP [4], Identifiers are used to state the information-centric communication model by utilizing the Publish-Subscribe Internet (PSI) model. Moreover, a separated forwarding identifier is used for transport routing and self-certifying is utilized for security. PSIRP uses flat name instead of hierarchical name as of NDN. Network-Inf is also a PSI and ICN-based paradigm, but content delivery is only conducted to the consenting Content routers to maintain host-independent communication and reduce undesired traffic.

Though there are various ICN-based models other than these mentioned three architectures, they are among the most promising ICN-based designs for the Future Internet (FI). Our proposal is mainly based on NDN prototype because NDN is considered as the only architecture among them that possesses the backward-compatible capability $[3,17]$. NDN also provides data integrity and authentication verification. Besides, NDN has a hourglass architecture with Content chunk layer as a "narrow waist" and top layers focus on streaming services rather than HTTP as in IP-based architecture. This matches the mobile users' growing content demand tendency of interactive services via their mobile devices, in case of transportation system.

In this paper, we state the public transportation system, e.g. train, bus for the case of ITS because thanks to positive characteristics including punctuality and convenience, public transportation vehicles are commonly used and getting more and more popular, especially in urban areas and big cities. The public transportation's commuters also have high tendency to use their mobile devices for different kinds of interested information/content via Internet during the period when they spend on public transportation system. Better still, the motion of a commuter can be predicted from the path of a train/bus line. In particular, the moving direction, stopping time at a specific station along with the moving time between two different stations can be pre-determined in normal cases (relatively fixed time-schedule). This means that connection time and disconnected time of a mobile node to a conventional wireless network in train system can be identified as the time when the train stops at a station and when it moves to another station, respectively. This feature is a great benefit to exploit proactive-caching scheme for transportation communication since the route is static and moving process can be predicted.

In order to meet commuter's demand for broadband service, it is inevitable to establish a standardized wireless access scheme for public transportation 
systems, especially in case passengers' desired interests are now mainly information centric services (e.g. HD movies, multimedia services) whereas the current network infrastructure of public transportation system still relies on traditional TCP/IP model.

This article focuses on both cost-efficiency (low energy consumption) and effectiveness perspective of wireless communication, hence we aim to address and solve the key problem spaces of ICN, namely: scalable, costefficient content distribution, mobility and disruption tolerance as identified in ITU Recommendation Y.3033 [20] for Data Aware Networking (DAN), which is considered as ITU concept of ICN for Future Internet. Besides, as motivated from mentioned transportation system feature, we propose to integrate proactive-caching based scheme utilizing ICN caching and naming functions for standardization process in ITS, to provide smooth access connection to valid content segment via 'smart scheduler', then enhance user experience. The simulation results corroborate our proposal efficiency by substantial diminishing the delay time, average number of hop together with improving data rate to support seamless wireless communications (thanks to our proactive caching strategy). In addition, the proposal can save about $20 \%$ energy consumption compared to conventional ICN model (NDN design). The proposed ICN model also acts as a solution for network scalability because it can help to relieve the network burden of cellular network (mobile network) by providing all commuters (wireless content users) fast access to large contents and high QoS content via their mobile devices (in essence, cellular network communication via Base Station has high cost and higher latency due to no caching capability and pre-caching scheme for moving content users).

Thus, we do believe that our proposal is a promising and feasible contribution for the ongoing ICN standardization process of major standardization bodies, which will lead to significant impact for the prevalent large-scale deployment of ICN toward goal of making it become our next Internet architecture in the near future.

\section{Proposed ICN Transportation Model Utilizing Proactive-Caching Based Mechanism}

In this section, we select the railway as a scenario of public transportation for ITS communication because nowadays, railway is a dominated public transportation system in the big metropolitan areas e.g. Tokyo, London and Moscow. 


\subsection{Communication Topology for Transportation System and Assumptions}

Our ICN system topology design for railway/train transportation communication system is built on top of NDN [3] as shown in Figure 1. We propose a 5-level tree based network topology comprising of a server as a root node and distinguished Content Routers (CRs) accompanying IEEE 802.11 Wireless Access Point (Wireless APs) allocated into remaining 4 levels of the tree (the central content router is at level 4). We assume that the ICN system consists of various contents and every content is stored at the server which acts as Content Provider (CP). Furthermore, all contents have same size and each ICN CR can cache same maximum number of contents. It can be seen from Figure 1 that we design ICN system topology of a prefecture with the idea that each prefecture has a central CR connected to the content server (CP) for ITS.

In this model, we assume one wireless AP, as a first-level CR, is equipped at each part (railroad car or wagon) of a train, to build a train-level Wireless Local Area Network (WLAN). A wireless AP can be connected to CRs at different stations, which act as level 2 routers. Considering wireless AP and other routers as NDN content routers, when an AP receives an Interest,

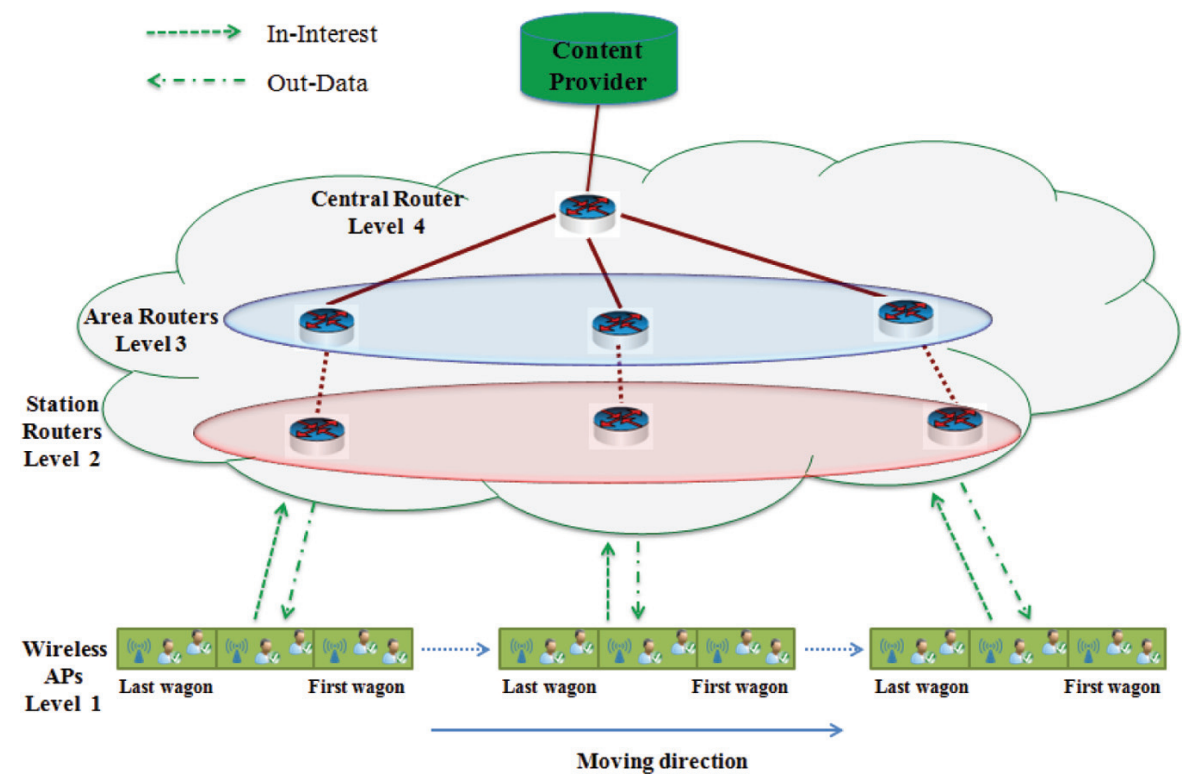

Figure 1 Proposed network topology for ITS. 
it first looks for the information object (content) in its content store (CS). If the content is found in CS, it sends back data to the content requester. If the content is not found in the CS, two tables i.e. Pending Interest Table (PIT) and forwarding information base (FIB) are used to handle the Interest packet. PIT collects incoming interfaces of the Interest packets so that the data packet can be delivered back on the same path (of incoming interest) towards the content requester whereas FIB directs Interest packet toward one or more content sources/repositories.

Hence, a commuter (mobile user) can connect to correspondent wireless AP of his/her current railroad car (Wifi) via connection between this AP and $\mathrm{CR}$ at a station when the train stop at a station. However, connection is not available during the moving periods of time. Due to the fact that the moving time is longer than the stopping time, the commuter is expected to endure the intermittent connection when connecting to wireless network of transportation system in current Internet architecture. CRs at stations (router level 2) are connected to higher level CRs including: Area (or city) CRs act as level 3 routers and prefecture (central) CRs act as level 4 routers via high-speed wired transmission.

When the train arrives and stops at a station, a wireless AP will get the precached content segments from CR of station via high speed wireless access based on proactive caching scheme. Consequently, the ratio of packet loss and latency in case of our proposal can be reduced compare to existing network system. Further detail of this proactive caching scheme and the way to divide a content into segments are clarified in the next part of this section.

Benefits of this network topology are easy deployment and practical design for the hierarchical structure of network and service provider in case of transportation industry. As a result, it is suitable for the migration process from current network infrastructure (with widely available and low-cost Wi-Fi APs). Besides, in this tree-topology, the more popular content is expected to be located closer to the end-user then offering lower latency and higher QoS for interactive mobile services.

\subsection{Proposed Proactive-Caching Based Scheme for Seamless Communication}

In this part, we describe proposed strategy of proactive caching to support seamless communication for mobile users in ICN based transportation system scenario. We select Aggregation points as the location of proactive caching to enhance the scope of sharing the content. For that reason, as can be seen from 
Figure 2, station routers act as Aggregation nodes (under the assumption that all the routers and wireless APs are CRs as defined in [3]). Let $C$ be the set of all content. When a mobile device first expresses its interest for a specific content $\mathrm{c}(\mathrm{c} \in \mathrm{C})$ to its current railroad car's wireless AP, the interest goes to the CP through wireless AP, then Aggregation node (current CR station) and respective higher level content routers (CRs at level 3 and level 4). Our goal is to populate the different segments of content on the en-route of the interest path as well as the disjoin-neighbour path. When the $\mathrm{CP}$ receives an interest asked for a content, that content data is divided into several segments and then these segments are pre-cached to a number of appropriate Aggregation nodes (station CRs at level 2). Size of segments of a specific content can be calculated considering a fact that a content is composed of a set of chunks (a chunk is a unit for caching and transmitting data).

Let $\mathrm{N}$ be the expected number of stations that one commuter stays on the train, then proposed system pre-caches content's segments to a total of $(\mathrm{N}-1)$ stations' CRs away from the first station location where the content request is sent to $\mathrm{CP}$, according to the moving direction of the train line. With this

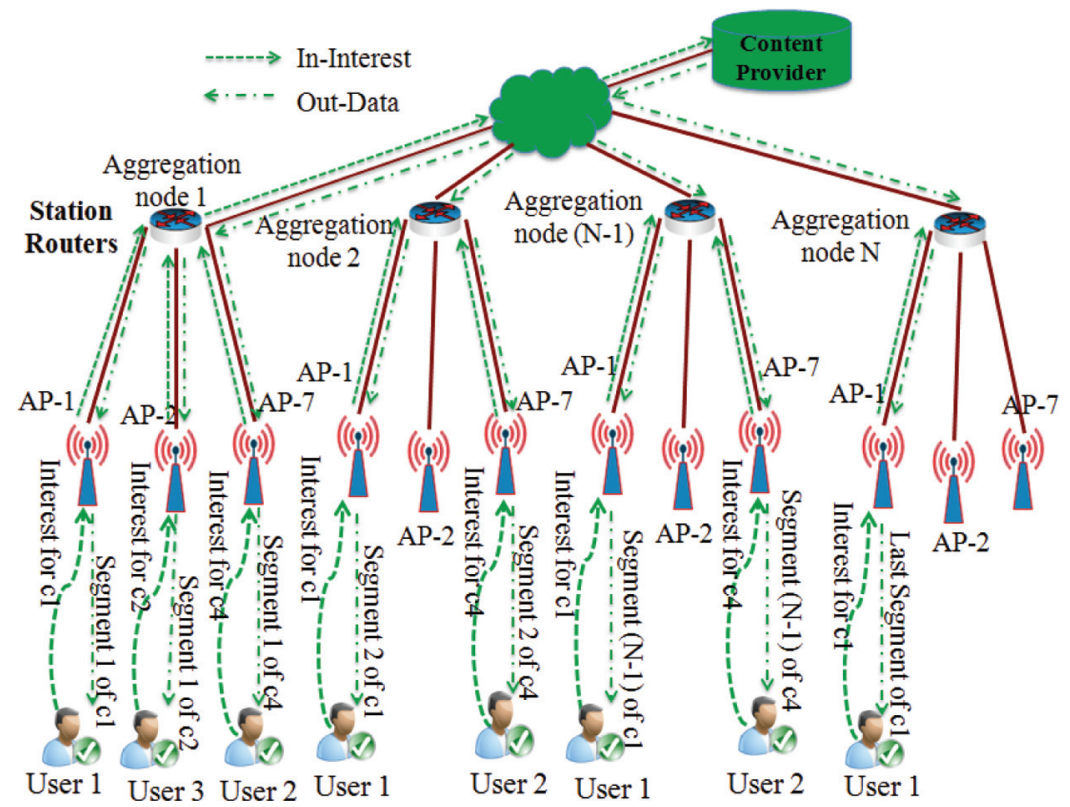

Figure 2 Flow of interest and content delivery path with proposed proactive caching in Station Router node for ITS network system. 
mechanism, a commuter is expected to get his/her full content within a total of $\mathrm{N}$ stations. The value of $\mathrm{N}$ is also used to identify the size of different segments of content will be pre-cached to different stations' CRs. A delivery scheduler, namely "smart scheduler", define the way how a content is precached to stations. This "smart scheduler" decides the appropriate location (station) for pre-caching by applying our proposed proactive caching strategy and can calculate the amount of content segment should be cached to maximize gain and user performance. Moreover, in order to prevent redundant content traffic, the pre-caching process for a suitable segment of a specific content $\mathrm{c} \in \mathrm{C}$ to station N's CR only happens in the case that station $(\mathrm{N}-1)$ still gets the interest for that content from same user at the time train stops at a station. Otherwise, the next segment is dropped, i.e. the time that commuter expresses his/her interest for a content at station $(\mathrm{N}-1)$ is the time the next segment of content is pre-cached to station N's CR (upcoming station of station $(\mathrm{N}-1)$ in train line). This is because next segment of the content is likely to be requested there in the next phase. In other words, if a commuter does not generate any interest for a content at a station (his/her expected station at one time) then segment of that content will not be pre-cached to the next station's $\mathrm{CR}$ to prevent possible congestion. This mechanism deals with the situation that a commuter leaves the train earlier than expected.

In order to do that, the system generates 'fake' interest (for same content) from the neighbor aggregation node (next/nearby station's CR). Thus, both aggregation points (en-route and out of route) fetch the content and cache the content. The cached segments in APs can be accessed by all matched subsequent mobile content users, then diminishes the network bandwidth consumption. Hence, when the train moves to the nearby station and user shows his/her interest for a content $c_{i}$ (content they already asked) again, the interest will be served via current railroad car's wireless AP from station CR because a valid segment of content is already cached in this CR. Figure 2 demonstrates the operating procedure of our proactive-caching based scheme in ICN for ITS through an example: At first, there are three users who send three interests for three different contents from three APs (i.e. three wagons of the train) at a specified station (aggregation node 1) on train line: user 1 sends interest for content $\mathrm{c}_{1}$ to wireless AP1, user 2 sends interest for content $\mathrm{c}_{4}$ to wireless AP7 and user 3 expresses interest for content $\mathrm{c}_{2}$ to wireless AP2. At that time, segment number 2 of these contents $\left(c_{1}, c_{2}\right.$ and $\left.c_{4}\right)$ will be calculated, determined and pre-cached to the aggregation 2 (nearby station). Then when the train moves to aggregation node 2 (nearby station), user 1 and user 2 continue asking for their interested contents so segment number 2 
of these contents will be served to them. Whereas user 3 stops sending his interest for content $c_{2}$ (he leaves the train at this station), hence the segment 3 of $\mathrm{c}_{2}$ will not be pre-cached to aggregation node 3 (next station). User 1 and user 2 continue sending their interests for content $\mathrm{c}_{1}$ and $\mathrm{c}_{4}$ to aggregation node $(\mathrm{N}-1)$, i.e. $(\mathrm{N}-2)$ station away from the first station (aggregation node 1$)$. However, only user 1 gets the full data of his interested content $c_{1}$ by sending interest for his desired content at aggregation node $\mathrm{N}$, and receiving the last segment of $\mathrm{c}_{1}$ from aggregation node $\mathrm{N}$ (user 2 and user 3 do not get the whole data they get interest because they leave the train earlier than expected). Thus, this proactive caching strategy provides a higher efficiency, better congestion control and reliability for the network system and mobile users. In addition, because we design the wireless AP for each railroad car of the train so during period that train moves between different stations, users also can get their interested data from the suitable wireless AP which acts as an ICN CR (after this AP gets appropriate data segment of interested Data from the station CR), then better support for the seamless connection.

Specifically, in our ICN system, a commuter (mobile content user) only sends interest for a specific content to the $\mathrm{CP}$ at first station by reason of our proposed proactive caching strategy whereas in conventional ICN design, a content request from a commuter needs to come to a server in the case that no content node contains that content. Worse still, getting data from server is the only way to retrieve a content/data in current IP-based network system. Hence, the delay will be diminished substantially since the interest does not need to go to CP. Thanks to this working mechanism, we show that the proposed prefetching protocol is relevant for the standardization process of ICN in context of ITS. This scheme also offers a reliable communication since information is firstly served by authorized and authenticated $\mathrm{CP}$ of service provider. Then suitable segments of contents can be transmitted to lower level nodes. Therefore, mobile users can get continuous content segments from appropriate AP via smart scheduler during period when they stay on train with expected

lower latency and shorter retrieval time. As a result, the proposed scheme provides higher QoE and better mobility performance for passenger as well.

\section{Adaptive Link Rate Operating Strategy for Greening ICN-Based ITS}

In this section, we state our proposed adaptive strategy of Content Nodes (CNs, including all CRs and Wireless APs) equipped with aforementioned ALR technique (some of which were introduced in our previous paper [11]). 
Firstly, we define $p_{k}$ as the probability that one content user can find a specific content $\mathrm{c} \in \mathrm{C}$ at a $\mathrm{CN}$ level $\mathrm{k}$, under consumption that all CNs located at the same level $\mathrm{k}$ of the tree network topology share the same value of $p_{k}$ because we assume network is symmetric and the requests generated by all users are homogeneous.

In case of current IP-based Internet architecture, we can infer that $p_{1}=$ $p_{2}=p_{3}=p_{4}=0$ and $p_{5}=1$ since as mentioned, different from ICN design, the only way to get a content/data is sending the request to server via routers. Moreover, more popular content is expected to be closer to the users because it is replicated more frequently compared to non-popular one. This means that the more popular a content is, the higher possible an user can find it at lower level, i.e. the popular contents have tendency to possess higher value of $p_{1}$ than the un-popular contents. Hence, we define two kinds of content:

$$
\begin{cases}\text { popular content: } & p_{1} \geq T_{p} \\ \text { non-popular content: } & p_{1}<T_{p}\end{cases}
$$

where $T_{P}$ as the threshold value of $p_{1}$ for all Content $\mathrm{c} \in \mathrm{C}$ (assume that all contents have same value of $T_{P}$ ).

Similar to $p_{k}$, the number of levels (hops) that content user traverse is expected to be lower with more popular content. Hence, the expected number of hops (levels) that user has to travel would be decreased. Let $Q_{k}$ is the probability that a content user traverses k-level (or k hops, where $\mathrm{k} \geq 1$ ) of the proposed tree topology to find an interested content $\mathrm{c} \in \mathrm{C}$, then $Q_{k}$ can be defined as following:

$$
Q_{k}=p_{k+1} \Pi_{l=1}^{k}\left(1-p_{l}\right)
$$

Then, we determine the dynamic operating power of ICN CRs to match their optimized utilizations by adjusting the correspondent link rate of CRs based on the popularity of the contents that the ICN nodes serve.

Let $R_{k}$ is the incoming link rate to a level-k CR for a content $\mathrm{c} \in \mathrm{C}$. Since a popular content has higher chance to be found at the first levels, the maximum link rate is set for the level $1 \mathrm{CRs}$ (Wireless APs) as in case of Conventional ICN:

$$
R_{1}=R_{I C N}
$$

where $R_{I C N}$ is the link rate enter a CR in Conventional ICN model. Let $S_{k}$ is the set of content interests come to a level k content router. Then $R_{k}$ (with $\mathrm{k}>1$ ) will adapt to the operating link utilization of ICN node based on popularity level of content $\mathrm{c}$ and the value of $R_{1}$ (for every interest for content c $\in S_{k}$ ):

$$
R_{1}>R_{k}(k>1)
$$


Since in general, one content node may receive various interests to ask for different contents, we consider two cases: One is Interest(s) come to a content node but only ask for un-popular content(s) and the other is there is at least one Interest sent to that node to ask for popular content. Then optimized value of $R_{k}$ for a level k-ICN router, namely Optimized $R_{\mathrm{k}, \mathrm{ICN}}(1<\mathrm{k} \leq 4)$, in case that there is at least one popular content is asked:

$$
\begin{array}{r}
\text { Optimized } R_{k, I C N}=\alpha\left\{R_{I C N}\left[1-\min \left(P_{1 c}+\sum_{l=1}^{k-2} Q_{l c}\right)\right]\right\} \\
\forall \text { Content } c \in S_{k} \text { and }\left|S_{k}\right| \leq S
\end{array}
$$

where $\alpha$ is the proportional coefficient of link rate and power consumption of Content Nodes (APs and CRs). $\alpha \geq 1$ and $\alpha=1$ means link rate is directly proportional to power consumption of network devices. In addition, $\mathrm{c}$ in the equation refers to all content(s) arrive to the CR level k.

and

$$
\text { Optimized } R_{k, I C N}=\alpha\left\{R_{I C N} \frac{\left.\frac{\max P_{1 c}}{T_{P}}\left[1-\min \left(P_{1 c}+\sum_{l=1}^{k-2} Q_{l c}\right)\right]\right\}}{\forall \text { Content } c \in S_{k} \text { and }\left|S_{k}\right| \leq S}\right.
$$

otherwise, i.e. user only expresses interest for unpopular content(s). The min function in Equations (4) and (5) returns the minimum value of argument for various values of c, i.e. it guarantees that the adapted link provides adequate utilization for the content with highest utilization request in Equation (4). Similarly, the max function in Equation (5) returns the maximum value of all arriving content $\mathrm{c}$ to enable enough link utilization for most popular content from all (unpopular) contents at that level. This mechanism assures that a $\mathrm{CN}$ can adapt its link rate to optimized utilization in order to satisfy all the interests for different contents (with different popularities) which were sent to it.

Since $\alpha$ may get value $>1$ then in case device is not fully support ALR function and value of Optimized $R_{k, I C N}$ indentified from Equation (4) or (5) is higher than $R_{I C N}$ (i.e. Optimized $R_{k, I C N}>R_{I C N}$ ) then let: Optimized $R_{k, I C N}=R_{I C N}$.

We then define the Power Adjustment Factor $\mathrm{P}_{\mathrm{A}}$ :

$$
P_{A}=\frac{\text { Optimized } R_{k, I C N}}{R_{k, I C N}}\left(0<P_{A} \leq 1\right)
$$

Let $\mathrm{P}_{\mathrm{R} 2-\mathrm{ICN}}$ be the operating power consumed by a content router in conventional ICN design (more detail in Section 6). Since we assume that all CRs are equipped with ALR function, the optimized value of operating power 
consumption of Content Node at level $\mathrm{k}$ in ICN (Optimized $P_{R 2-I C N, k}$ ) can be identified as:

$$
\text { Optimized } P_{R 2-I C N, k}=P_{A} P_{R 2-I C N}
$$

Therefore, for this ICN proposal, when a content gets more popular then the load of the network decreases and diminishes the transport energy notably.

\section{Mathematical Energy Models for Energy Efficiency Analysis}

The total energy of network system can be considered as sum of the energy consumed by all network components and devices that make up the network system.

For scope of our research, we do not consider the overhead power consumption of network, e.g. cooling and lighting energy, and assume that each network system comprises two major elements: $N$ content nodes (CRs and APs) and one server (CP). In this way, we present the respective mathematical models for energy consumption of current IP-based network and conventional ICN design, referred to [21]. Then, for energy-efficiency evaluation, we take into consideration the factors of network topology, content popularity, network device energy efficiency capability to build our proposed Green ICN system model for energy savings evaluation. This section states our in-depth energy evaluation models with further extensive analysis from our previous work [11].

\subsection{IP-Based Network System Energy Consumption}

The energy consumed by a IP-based system (traditional TCP/IP model) can be expressed as:

$$
\begin{aligned}
& E_{I P}=N E_{R-I P}+E_{S}=N P_{R 1-I P} T_{W}+N_{1} P_{R 2-I P} T_{W} \\
& \quad+N_{2} P_{R 2, A P-I P} T_{W}+\left(P_{S 1} T_{W}+P_{S 2} T_{W}+P_{S 3} T_{W}\right)
\end{aligned}
$$

where $E_{R-I P}, E_{S}$ are the energy consumed by a IP router and energy consumed by the server; $P_{R 1-I P}, P_{R 2-I P}, P_{R 2, A P-I P}$ are the embodied power of a network node (router/AP), working power of a IP router, and working power of an AP, respectively; $N_{1}, N_{2}$ and $\mathrm{N}$ are the number of routers, number of APs, and number of CNs respectively $\left(N_{1}+N_{2}=N\right)$ and $P_{S 1}, P_{S 2}, P_{S 3}$ are the embodied power, power for server storage and operating power of a 
server (same value for both ICN and IP based network system), respectively. Besides, $T_{w}$ is the working time of the whole network system.

\subsection{Conventional ICN System Energy Consumption}

The conventional ICN system energy consumption can be calculated as:

$$
\begin{array}{r}
E_{I C N}=N E_{R-I C N}+E_{S}=N\left(P_{R 1-I C N} T_{W}+P_{R 3-I C N} T_{W}\right) \\
+N_{1} P_{R 2-I C N} T_{W}+N_{2} P_{R 2-I C N, A P} T_{W} \\
+\left(P_{S 1} T_{W}+P_{S 2} T_{W}+P_{S 3} T_{W}\right)
\end{array}
$$

where $P_{R 1-I C N}, P_{R 2-I C N}, P_{R 3-I C N}$ are the embodied power, working power and power to cache memory of a ICN CN (CR/AP), respectively. For the purpose of power consumption evaluation, both the current IP-based network system and conventional ICN system share the same power consumption for servers, whereas a ICN node consumes slightly higher power compared to a normal IP node because of the CN's caching function (additional cache memory energy for CR in case of ICN).

\subsection{Proposed Green ICN Model for ITS Energy Consumption}

The optimized value of total energy consumed by our proposed Green ICN system is a combination of two optimized values:

$$
\begin{aligned}
& \text { Proposal } E_{I C N}=\sum_{k=1}^{N} \text { Optimized } E_{R-I C N, r k} \\
& + \text { Optimized } E_{S-I C N}
\end{aligned}
$$

where optimized energy consumption of all CNs:

$$
\begin{aligned}
\sum_{k=1}^{N} \text { Optimized } E_{R-I C N, r k} & =N\left(P_{R 1-I C N} T_{W}+P_{R 3-I C N} T_{W}\right) \\
+ & \sum_{k=1}^{N} \text { Optimized } P_{R 2-I C N, r k} T_{O r_{k}}(11)
\end{aligned}
$$

and optimized value of server $(\mathrm{CP})$ :

$$
\begin{aligned}
& \text { Optimized } E_{S-I C N}=\left(P_{S 1} T_{W}+P_{S 2} T_{W}\right) \\
& +\left[P_{F} T_{O s}+P_{1}\left(T_{W}-T_{O s}\right)\right]
\end{aligned}
$$

where $T_{O r_{k}}$ is the operating time of $\mathrm{CN} r_{k}$ with proposed ALR design, and $T_{O s}$ is the operating time of server $\mathrm{S}$. Besides, assume that systems uses server (CP) 
with two specific states: Idle mode when no content interest send to server and Full mode otherwise (there is at least one interest come to CP during a period time T). Then let $P_{F}$ and $P_{I}$ are working power of Full mode and Idle mode and assume that $P_{I}=0.3 P_{F}$.

\section{Performance Evaluation and Discussion}

In this section, we verify benefits of proposed greening mechanism together with proactive caching strategy in ICN architecture to enhance the user experience of the mobile users in case of commuter train's passengers.

We simulate our proposed ICN based system in ITS with ndnSIM, which is a scalable emulator of Named Data Networking (NDN) [22] under the NS-3 framework, to evaluate efficiency of proposed wireless access ICN based scheme for public transportation in contrast with traditional TCP/IP and Conventional ICN model (NDN design). The network topology used in the simulation is tree topology as aforementioned: There are four ICN CRs level 3 (area/city CRs), four CRs act as ICN station CRS (CRs level 2) and the prefecture/central CR at level 4 is connected to the repository (server/content provider) to form a 5-level tree network topology.

We assume that a train has seven distinguished railroad cars, and each car has its own dedicated wireless AP. There are two commuters (mobile content users) at each railroad car and a mobile content user/client (passenger on train) is connected to respective wireless AP level 1 . He/she first demands for a specific content via mobile devices, i.e. sends Interest packet for that content and an ICN content server acts as content provider for providing content. The content is then proactively cached at every train station according to the "smart scheduler", which decides the appropriate amount of content segment should be proactively cached (pre-cached) in ICN routers of train stations and the delivery schedule including location and timing delivery as described in Section 4.

The period of time for staying at each station and moving between two stations are 18s and 90s, respectively. The stoppage time at each station is 18 seconds, which can be determined as an average value for an train line in practice. For simplicity, we take $\mathrm{N}$ (expected number of stations that a user stays on a train line) equal to four. Wireless APs are connected via IEEE 802.11g standard. Assume all the ICN nodes have the functionalities of PIT (Pending Interest Table), FIB (Forwarding Information Base) and CS (Content Store) as described in [3]. Total number of objects/different contents is 20,000 and assume that a content user does not generate any interest for the 
objects/contents which are not stored in the repository. The Zipf distribution, which is similar to the Zipf-like distribution as defined in [23] is used for the content popularity distribution. Content request rate is equivalent to $25 \%$ of network utilization. Cache object eviction policy is LRU (Least Recently Used). We also assume that every content has the same size of $1000 \mathrm{MB}$ (with payload size of $1 \mathrm{~KB}$ ). The link capacity/bandwidth we use for simulation is 1 Gbps. The network elements and their respective power consumptions for evaluation are referred to data presented in [21, 24]. Under the assumption that we have two similar network systems with same characteristics: one follows conventional ICN (NDN design) and the other has traditional IPbased architecture. We then make simulation and demonstrate our proposal performance and efficiency compared with these two existing network system designs, in terms of hop count, network delay, Interest Data rate and energy consumption with the above parameters as following:

- Average Packet Hop count of proposed ITS system: the Average Packet Hop count is almost stable with the simulation time as showed in the Figure 3 except the cases that the Mobile Node (MN) is involved in the Hand-offs period when it moves to change the PoA to another Station node (when train stops at a station). The higher value for this metric

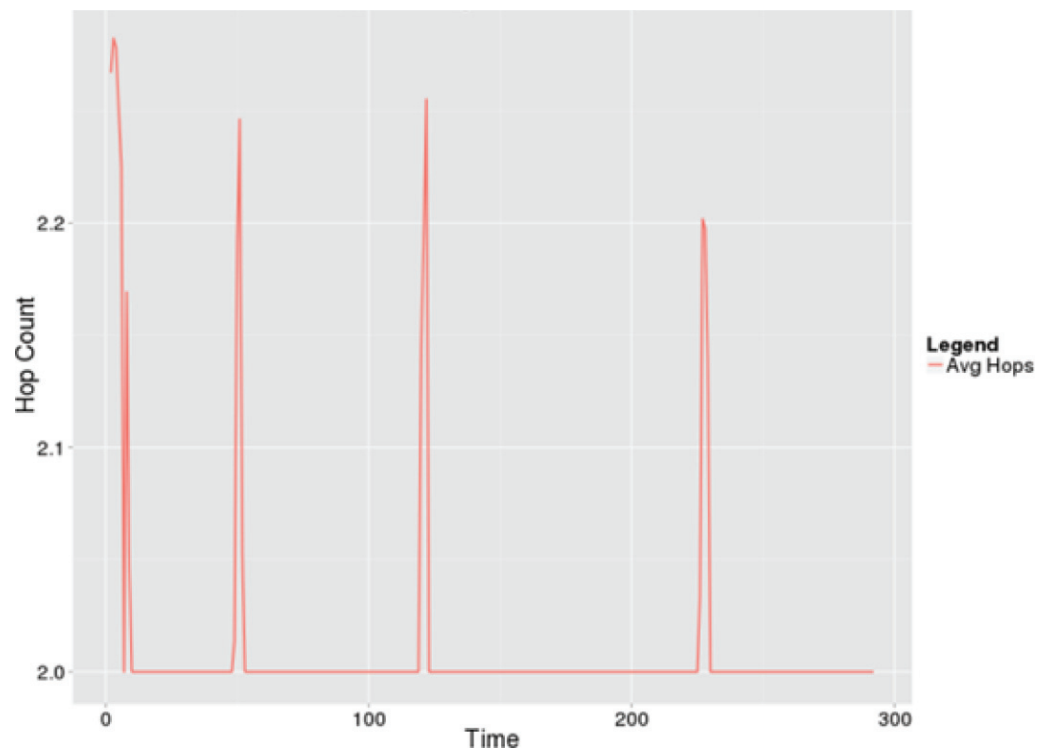

Figure 3 Performance evaluation for Average Hop Count of proposed ITS network system. 
represents the case of hand-off. And as we can see from the figure that the period taken to hand-off is very short, thanks to our "smart scheduler" and innovated proactive caching strategy.

- Average Network Delay of proposed ITS system: For the objective of seamless mobile communication, network delay is a key metric. From our simulation result as shown in Figure 4, similar to the Average Packet Hop, the average delay is kept at less than 6 millisecond which is considerably very low. Even in the hand-offs period when the mobile content user moves to change the PoA from one station to another station (Wireless AP node), the delay is less than 10 millisecond. It can be seen that a mobile user can get a smooth connection with almost no disruption during the time he/she is on train.

- Interest Data Rate of proposed ITS system: For this metric, we evaluate the rate of both Data packet and Interest packet, in case that the train moves at different speeds and proposed ICN model for ITS utilizing our proactive-caching strategy or not. It can be seen from the Figure 5 that the Interest rate does not directly affected by speed. Instead of that, it maintains a stable rate with or without proposed proactivecaching scheme. On the other hand, though as expected, when the train

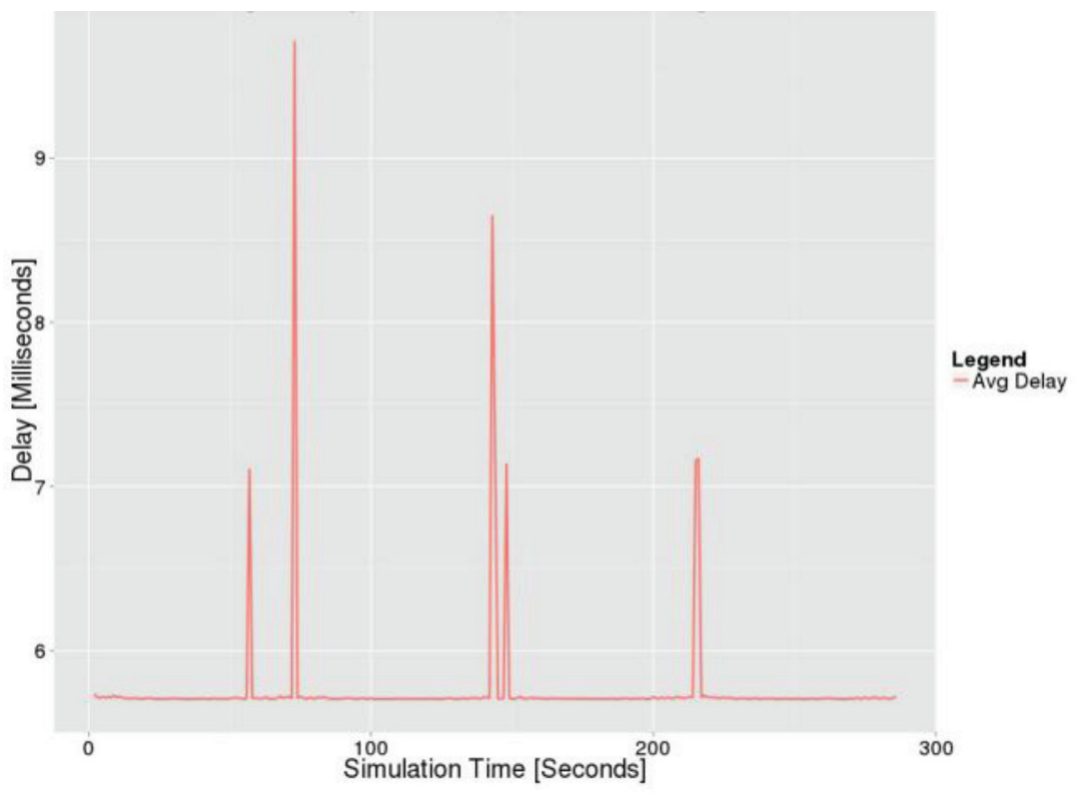

Figure 4 Performance evaluation for Average Network Delay of proposed ITS network system. 


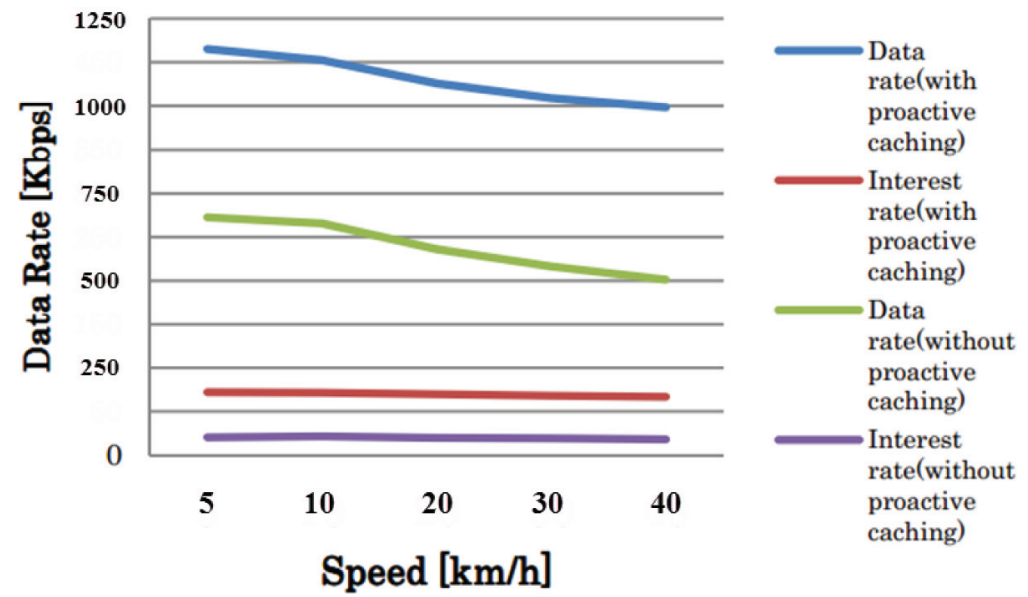

Figure 5 Performance evaluation for Interest Data Rate versus train speed of proposed ITS network system.

starts moving faster, data rate is lowered in both case, the proposed proactive caching with "smart scheduler" offers data rates about double compared to the case of proactive caching absence. This is because using proposed proactive-caching scheme, passengers can get valid segments of interested contents that are previous requested in the Content Store of neighbour Station Routers during the time they stay on train. Hence, we show that the proposal provides better performance for commuter to gain seamless content access while moving by significant reducing retrieval time.

Note that within scope of this article, we do not consider similar prefetching scheme of proposed model for other wireless systems. The detail evaluations, analyses and comparisons between our system and other wireless systems utilizing proactive-caching scheme for moving vehicles will be conducted and stated in another work.

- Impact of ICN CN caching size on average power consumption: From our simulation result as shown in Figure 6, the average power consumption of both Conventional ICN and our proposed ICN model increase when we increase the size of the content cache of each $\mathrm{CN}$ (five different ICN CN cache sizes: $64 \mathrm{~GB}, 96 \mathrm{~GB}, 128 \mathrm{~GB}, 192 \mathrm{~GB}$ and $256 \mathrm{~GB}$ with $\alpha=1$ ). This is because ICN system need to endure additional caching energy for the $\mathrm{CN}$ as stated in Sub-section 6.2 (the respective values of CNs' 


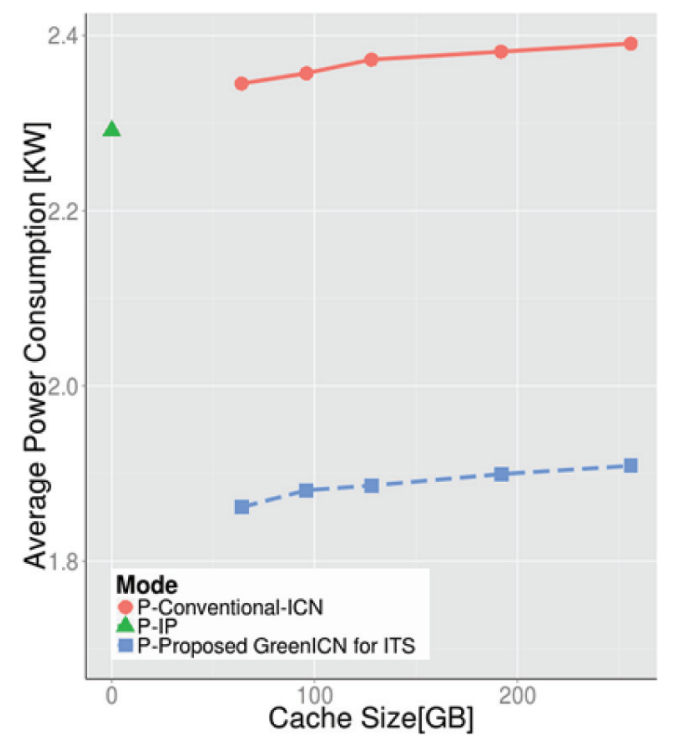

Figure 6 Average power consumption of different network systems versus the different cache size of ICN content router.

power consumptions can be found in [21]). Moreover, proposed model can save significantly power consumption compared to other current network designs.

- Impact of Alpha value $(\alpha)$ on average power: As can be seen in Figure 7, the value of $\alpha$ and average power consumption of the network system have a linear relationship. Therefore, when we increase the value of $\alpha$, as expected, the energy consumption of the proposed ICN system is also increased. Typically, we take value of $\alpha$ ranges from 1 to 1.3 for the evaluation. Though Conventional ICN consumes slightly higher energy consumption compared to our current IP-based architecture due to additional energy for caching capability, from Figure 7, our proposed Green ITS model can substantially decrease energy consumption. In more detail with $\alpha=1$ (the ideal case with ALR-fully support $\mathrm{CN}$ ), the proposed system can save about $21.16 \%$ energy compared to conventional ICN in the same scenario, whereas this ratios are decreased to $18.57 \%$ with $\alpha=1.3$.

In general, for all of the discussed metrics, simulation with topology for ITS proves the efficiency of our proposal proactive caching ICN model compared to current IP-based model and Conventional ICN design, in terms of energy 


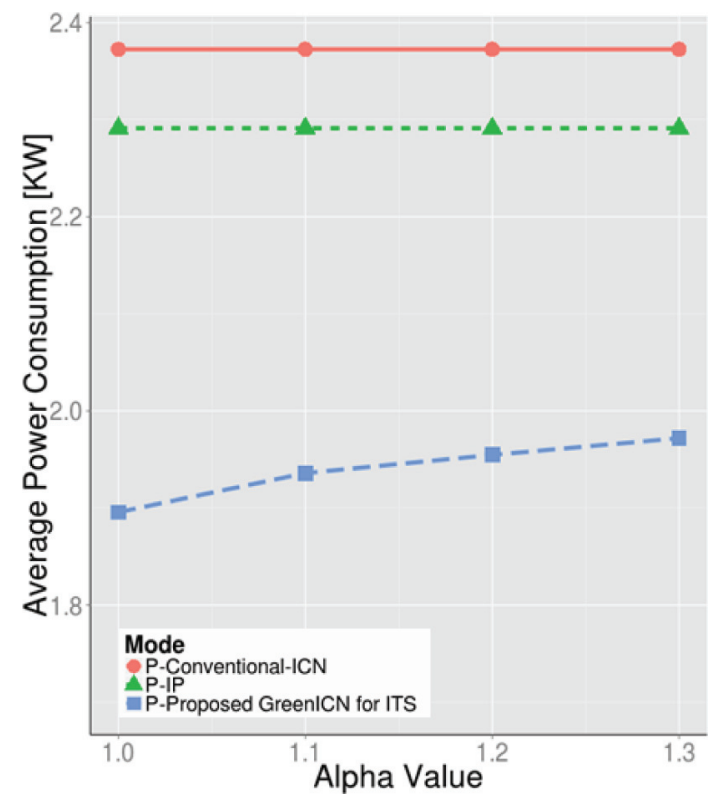

Figure 7 Average power consumption of network systems versus $\alpha$ value.

efficiency and effectiveness (by utilizing flexible and efficient integrated solution of combining ALR and proactive caching based schemes).

\section{Conclusion and Future Work}

It is undoubted that ICN Architecture, which based on named data networking $[1,2]$ to disseminate information effectively instead of host name, is highly efficient compared with our existing IP-based Internet Architecture. In this article, we have designed an ICN communication model utilizing innovated dynamic pre-fetching and ALR based strategy together as a practical ITS solution to offer reliable and effective wireless content access for the commuters in transportation system (particularly public transportation systems). Thanks to the "smart scheduler", appropriate content segments are pushed in advance, then the proposed ICN-based Internet access scheme can significant diminish negative impacts, e.g. high latency, low data rate and support seamless communication by improving reliability, QoE and network performance. The simulation results corroborate our theoretical idea and reveal the efficiency of our proposed scheme, in terms of effectiveness (low latency) and energy 
efficiency (up to $20 \%$ energy saving), compared to both of current IP-based Internet architecture and conventional ICN (NDN design).

Furthermore, by showing the significance of setting up the standardization of ICN wireless communication in the transportation system perspective, we propose our work for official standardization process of major standardization bodies, e.g. ITU, IETF and IRTF. We do believe that this proposed ICN-based Internet access model for ITS is a promising and feasible contribution for the standardization process of ICN by integrating Green networking into ICN to combine the benefits of innovated rate-adaptivity and proactive-caching based schemes for mobile content access, in case of public transportation's content users. It is also a potential pioneer solution to future standard and ICN standardization process for utilizing in transportation industry with simple and practical deployment of tree-based topology for both network and service provider (transportation industry) by taking advantage of low-cost and widely available feature of Wi-Fi. Besides, the proposal fits our future-looking scope of trust information infrastructure and energy management for digital ecosystem to build a reliable and safe human-centric network system toward an ubiquitous intelligent and trusted information society.

For the scope of future work, the realization of the scheme under field experiment with larger scalability (multiple mobile routers are deployed on train/bus with spatial wireless signal diversity) as well as simulation for various and practical use-cases (e.g. identifying high content traffic period of time when mobile users generates much higher interest demand during rush-hours, compared to normal or low content traffic of remaining periods during one day in case of transportation system) with distinguished kinds of content services and applications, including VoIP, HD Multimedia services, etc. are needed to further evaluate proposal's efficiency. We also plan to take the train experiment data to find an optimal location for Wireless APs of a train's railroad car in different scenarios e.g. the position together with mobility of commuters in each railroad car and different number of mobile content users including user arrival rate. Since network condition should reflect the change of commuter numbers at different stations when new mobile users enter whereas the current content users may leave the transportation system. Last but not least, in order to confirm the efficiency of proposed ICN model and raise proposal potential for international standard, a more detailed description, evaluation and analysis of the proposed model comparing with other wireless technologies and alternate mobile communication standards which also support caching and pre-fetching at Base Stations will be carried out for our future work. 


\section{References}

[1] Jacobson, V., Mosko, M., Smetters, D., and Garcia-Luna-Aceves, J. J. (2007). Content-centric networking: Whitepaper describing future assurable global networks. Response to DARPA RFI SN07-12.

[2] Trossen, D., and Parisis, G. (2012). "Designing and realizing an information-centric internet", Proceeding of the IEEE Communication Magazine, vol. 50.

[3] Jacobson, V., Smetters, D. K., Thornton, J. D., Plass, M. F., Briggs, N. H., and Braynard, R. L., (2009). "Networking named content", in Proceeding of the 5th International Conference on Emerging Networking Experiments and Technologies (CoNEXT '09). ACM: Rome, 1-12.

[4] Gaddah,A., and Kunz, T. (2010). Extending mobility to publish/subscribe systems using a pro-active caching approach. Mob. Inf. Syst. 6, 293-324.

[5] Pack, S., Jung, H., Kwon, T., and Choi, Y., (2005). SNC: a selective neighbor caching scheme for fast handoff in IEEE 802.11 wireless networks. ACM Mob. Comput. Commun. Rev. 9, 39-49.

[6] Vasilakos, X., et al. (2012). Proactive selective neighbor caching for enhancing mobility support in information-centric networks. Proc. ACM ICN 12, 61-66.

[7] Gunaratne, C., et al. (2008). Reducing the energy consumption of ethernet with Adaptive Link Rate (ALR). IEEE Trans. Comp. 57, 448-461.

[8] Zhang, B., Sabhanatarajan, K., Gordon-Ross, A., and George, A. (2008). Real-time performance analysis of adaptive link rate, in Proceeding of the 33rd IEEE Conference on Local Computer Networks, Montreal, QC, 282-288.

[9] Bilal, K., et al., (2013). A survey on green communications using adaptive link rate. Cluster Comput. 16, 575-589.

[10] Nedevschi, S., et al., (2008). "Reducing network energy consumption via sleeping and Rate-Adaptation," in Proceeding of the 5th USENIX Symposium on Networked Systems Design and Implementation (NDSI'08), Berkeley, CA, 323-336.

[11] Nguyen, Q. N., Arifuzzaman, M., Miyamoto, and Takuro, S. (2015). "An optimal information centric networking model for the future green network," in Proceeding of the IEEE Twelfth International Symposium on Autonomous Decentralized Systems (ISADS), Taichung, 272-277. 
[12] P. Rodiguez, et. al. (2004). "MAR: a commuter router infrastructure for the mobile internet," in Proceedings of the 2nd International Conference on Mobile Systems, Applications, and Services, Boston, MA, 217-230.

[13] Kumar, K. R., Angolkar, P., and Das, D. (2008). "SWiFT: a novel architecture for seamless wireless internet for fast trains," in Proceeding IEEE Vehicular Technology Conference, (Nanjing: VTC spring), 3011-3015.

[14] Tso, F., Cui, L., Zhang, L., Jia, W., Yao, D., Teng, J., and Xuan, D. (2013). "DragonNet: a robust mobile internet service system for long-distance trains," in Proceeding of the IEEE Transactions Mobile Computing, Vol. 12, Piscataway, NJ, 2206-2218.

[15] Amadeo, A., Molinaro, M., and Ruggeri, G. (2013). E-CHANET: Routing, forwarding and transport in Information-Centric multihop wireless networks. Comput. Commun. 36, 792-803.

[16] Nguyen, Q. N., Arifuzzaman, M., and Sato, T. (2015). Proactive-caching based information centric networking architecture for reliable green communication in intelligent transport system," in Proceedings of the 2015 ITU Kaleidoscope: Trust in the Information Society (K-2015), Barcelona, 1-7.

[17] Zhang, L., Estrin, D., Burke, J., Jacobson, V., Thornton, J., Smetters, D., et al. (2010). Named Data Networking (NDN) Project. PARC Technical Report NDN-001.

[18] Trossen, D., (ed.) (2009). Architecture Definition, Component Descriptions, and Requirements. Available at: http://www.psirp.org

[19] Ahlgren, B., et al. (2010). Second NetInf Architecture Description. Available at: http://www.4ward-project.eu/

[20] ITU-T. (2014). Recommendation ITU-T Y.3033: Framework of Data Aware Networking for Future Networks. Available at: http://handle.itu.int/ $11.1002 / 1000 / 12076$

[21] Butt, M. R., Delgado, O., Coates, M. (2012). "An energy-efficiency assessment of Content Centric Networking (CCN)," Electrical \& Computer Engineering (CCECE), Montreal, QC.

[22] ndnSIM homepage, Available at: http://www.ndnsim.net/

[23] Breslau, L., Cao, P., Fan, L., Phillips, G., and Shenker, S. (1999). Web Caching and Zipf-like Distributions: Evidence and Implications. In Proceedings of the IEEE Conference on Computer Communications INFOCOM. Washington: IEEE Press. 
[24] Novarum Inc. (2009). Enterprise 801.11n Wireless Access Point Performance Benchmark. Available at: http://www.novarum.com/documents/ Enterprise802.11nSingleAPBenchmarkTestingv1.3.pdf

[25] GreenICN Project. (2013). Available at: http://greenicn.org/

\section{Acknowledgement}

This work has been supported by the EU-JAPAN Green ICN project initiative by the EC Seventh Frame-work Programme (FP7/2007-2013) Grant Agreement No.608518 (GreenICN) and NICT Contract No. 167.

\section{Biographies}

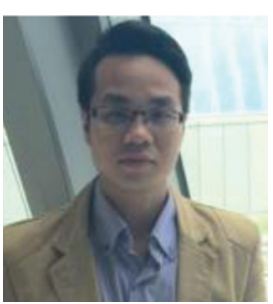

Q. N. Nguyen was born in Ha Noi, Vietnam. He received the B.E degree in Information Technology, Honor Computer Science Program conducted in English from Posts and Telecommunications Institute of Technology (PTIT), Ha Noi, Vietnam in 2012. After that, he was asked to stay to work at PTIT and became one of the youngest Faculty members of Institute. He was involved in building document and designing the curriculum for opening new major in Information Security, which is the first and pilot Regular Undergraduate program in Information Security in Vietnamese education. He was the sole Awardee of Asia Special Scholarship, Waseda University for Fall 2013 admission to Graduate School of Global Information and Telecommunication Studies (GITS), Waseda University, Tokyo, Japan. He graduated M.S. degree in Computer Systems and Network Engineering Area from GITS, Waseda University and currently, he is a Ph.D. Candidate at Graduate School of Fundamental Science and Engineering, Faculty of Science and Engineering, Waseda University. His research interests include Future Internet Architecture, Green Networking, Information Centric Networking and Next Generation Mobile Communication Systems. 


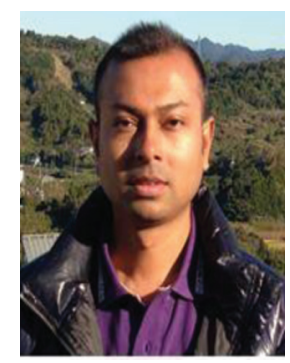

M. Arifuzzaman received B.Sc. degree in Computer Science \& Engineering from Bangladesh University of Engineering \& Technology (BUET) in 2001. He worked as an Assistant professor at IBAIS University, Dhaka, Bangladesh from 2001 to 2005. After that he joined in Bangladesh Civil Service in 2006 and worked as an Assistant secretary to the Government of the People's Republic of Bangladesh till 2010. He has completed Masters in Global Information and Telecommunication Studies, from Waseda University, Tokyo, Japan in 2012. He completed his Ph.D. in Global Information and Telecommunication Studies from Waseda University in 2015. He worked as a postdoctoral fellow at the Faculty of Engineering and Applied Science, Memorial University of Newfoundland, NL, Canada. Currently, he is an Assistant Professor at Department of Electronics and Communications Engineering at East West University, Bangladesh. His research interests lie in the area of Communication Protocols, Energy Efficient Networking, Future Network Architecture, Information-Centric Networking, Wireless Ad-hoc and Sensor networks, IoT, and Mobile Communications.

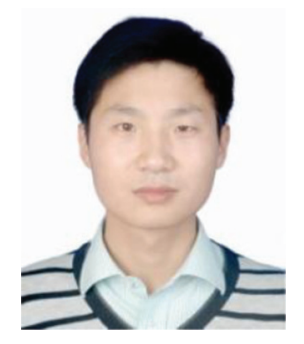

Di Zhang received his B. Eng. degree from the PLA Information Engineering University (2006-2010), Zhengzhou, China, M. Eng. degree (Distinction first honors) from Central China Normal University (2010-2013), Wuhan, China. He visited Advanced Communication Technology Lab, National Chung Hsing University, Taichung, Taiwan as a research student in 2012. Now he is a Ph.D. 
candidate in Waseda University, Tokyo, Japan. He served as the reviewer of IEEE Vehicular Technology Magazine (VT Mag.), IEEE VTC 2015 Spring and IEEE ISADS 2015, etc. He was granted the Best Master Thesis, Best Graduate, China Scholarship Council (CSC) Scholarship, etc. His research interests include green communications, massive MIMO, wireless communications and optical communications. He is a student member of IEEE and IEICE.

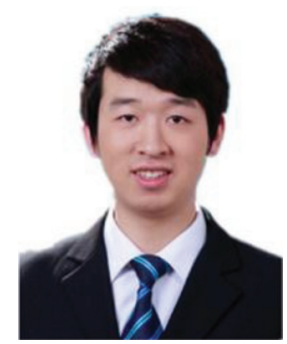

K. Yu was born in China, on January 1988. He received his B.E. and B.Admin. degree from Sichuan Normal University, Sichuan, China in 2010 and University of Electronic Science and Technology of China, Sichuan, China in 2010, respectively. He received his M.Sc. degree in Wireless Communication from Waseda University, Tokyo, Japan in 2012. Currently, he is a Ph.D. candidate at Graduate School of Global Information and Telecommunication Studies (GITS), Waseda University, Tokyo, Japan. He is an IEEE student member. His research interests include smart grid, ICN/CCN and their information security.

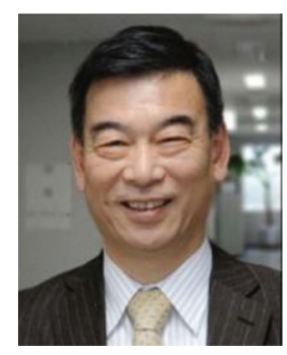

T. Sato received the B.E. and Ph.D. degrees in electronics engineering from Niigata University, Niigata, Japan, in 1973 and 1993, respectively. He joined the Research and Development Laboratories, OKI Electric Industry Co., Ltd., Tokyo, Japan, in 1973, where he was a Senior Research Manager and Research 
Director with Communication Systems Laboratory. He joined the Niigata Institute of Technology from 1995 as a Professor where his researches focused on CDMA, OFDM, personal communication systems and other related areas. In 2004, he joined GITS, Waseda University, Tokyo, as a professor and currently serving as the Dean. He has engaged in the research on PCM transmission equipment, mobile communications, data transmission technology, and digital signal processing technology. He has developed wideband CDMA system for personal communication system and joined the PCS standardization committee in the USA and Japan. His contributes are mainly in high speed cellular modem standardization for ITU, $2.4 \mathrm{GHz}$ PCS for ITA and wireless LAN technology for IEEE 802.11, etc. He has authored 11 Books and more than 200 papers. His current research interests include Next generation mobile communications, wireless communications, ICN technology, ICT in Smart Grid and their global standardization. He is a fellow of IEEE and IEICE. 